\title{
The X-ray spectrum of the Seyfert I galaxy Mrk 766: Dusty Warm Absorber or Relativistic Emission Lines?
}

\author{
K. O. Mason ${ }^{1}$, G. Branduardi-Raymont ${ }^{1}$, P. M. Ogle ${ }^{2}$, M. J. Page ${ }^{1}$, E. M. Puchnarewicz ${ }^{1}$, \\ E. Behar ${ }^{3}$, F. A. Córdova ${ }^{2}$, S. Davis ${ }^{2}$, L. Maraschi ${ }^{4}$, I. M. McHardy ${ }^{5}$, P. T. O’Brien ${ }^{6}$, W. C. \\ Priedhorsky $^{7} \&$ T.P. Sasseen ${ }^{2}$ \\ ${ }^{1}$ Mullard Space Science Laboratory, Department of Space and Climate Physics, \\ University College London, Holmbury St Mary, Dorking, Surrey, RH5 6NT, UK. \\ 2 Department of Physics, University of California, Santa Barbara, CA 93106, USA \\ 3 Columbia Astrophysics Laboratory, Columbia University, $550 \mathrm{~W} 120$ St, New York, NY \\ 10027, USA \\ 4 Osservatorio di Brera, via Brera 28, 20121 Milano, Italy \\ 5 University of Southampton, University Road, Southampton \\ ${ }^{6}$ Physics and Astronomy Dept., University of Leicester, University Road, Leicester, UK \\ 7 Los Alamos National Laboratory, Los Alamos, NM 87545, USA \\ kom@mssl.ucl.ac.uk
}

\begin{abstract}
Competing models for broad spectral features in the soft X-ray spectrum of the Seyfert I galaxy Mrk 766 are tested against data from a 130 ks XMM-Newton observation. A model including relativistically broadened Ly $\alpha$ emission lines of O VIII, N VII and C VI is a better fit to $0.3-2 \mathrm{keV}$ XMM-RGS data than a dusty warm absorber. Moreover, the measured depth of neutral iron absorption lines in the spectrum is inconsistent with the magnitude of the iron edge required to produce the continuum break at $17-18 \AA$ in the dusty warm absorber model. The relativistic emission line model can reproduce the broad-band (0.1-12 keV) XMM-EPIC data with the addition of a fourth line to represent emission from ionized iron at $6.7 \mathrm{keV}$ and an excess due to reflection at energies above the iron line. The profile of the $6.7 \mathrm{keV}$ iron line is consistent with that measured for the low energy lines. There is evidence in the RGS data at the $3 \sigma$ level for spectral features that vary with source flux. The covering fraction of warm absorber gas is estimated to be $12 \%$. Iron in the warm absorber is found to be overabundant with respect to $\mathrm{CNO}$ compared to solar values.
\end{abstract}

Subject headings: accretion, accretion disks - line: profiles - galaxies: Seyfert galaxies: individual(Mrk766) - X-rays: galaxies 


\section{Introduction}

The combination of the Reflection Grating Spectrometer (RGS) and the high throughput of XMM-Newton constitutes a powerful tool for probing the soft X-ray spectrum of bright AGN. The increase in spectral resolution has already caused a revision in thinking and has revealed a complex spectrum of narrow absorption lines arising in material with a range of ionisation states (e.g. IRAS 13349+2438; Sako et al. 2001). The XMM-RGS spectra of the Seyfert galaxies MGC-6-30-15 and Mrk 766 show evidence of broad spectral features (Branduardi-Raymont et al. 2001; hereafter BR01; see also Sako et al. 2002) and a similar spectrum of MCG-6-30-15 has been recorded using the grating spectrometers on the Chandra observatory (Lee et al. 2001). The nature of these features is controversial. Two competing models are: (1) absorption edges imprinted on the underlying spectrum by a dusty, warm absorber (Lee et al. 2001), (2) emission lines of O VIII, N VII and C VI broadened by relativistic motion in an accretion disk around a massive spinning black hole (BR01).

In this paper we discuss the first results of a long (130 ksec) XMM-Newton observation of Mrk 766. This has better statistics than the shorter observation of BR01. We examine how well the competing models are able to reproduce the RGS data. The way in which the spectral features change with source flux is investigated by comparing the mean spectrum taken during flaring episodes with that from intervals between flares. We also discuss the nature of the warm absorber. The spectrum in the full X-ray energy range of XMM-Newton is investigated, combining the RGS data with those recorded at the same time by the EPIC CCD spectral imager. We compare the low energy spectral features to the $\sim 6.7 \mathrm{keV}$ iron line detected using EPIC.

\section{Observations and Data Reduction}

Mrk 766 was observed with all the XMM-Newton instruments for a continuous period of $130 \mathrm{ksec}$ beginning 2001 May 21. This is essentially the maximum observing time possible during an XMM-Newton orbit. The RGS was used in standard spectroscopy mode (den Herder 2001). The EPIC MOS-1 camera was used in timing mode while MOS-2 and the PN camera were set to small window mode (Turner et al. 2001; Strueder et al. 2001). The OM instrument (Mason et al. 2001) took a series of ultraviolet images of Mrk 766 in various filters with a cadence of 1300 sec.

The data were reduced using the standard XMM-Newton Science Analysis Software. In this letter we consider the RGS and MOS-2 EPIC data only, the latter because the camera and operating mode combination currently has the best established calibration among those 
used.

\section{Analysis and Results}

We detect X-ray variations in Mrk 766 of up to a factor of two on a timescale of a few thousand seconds. The light curve of the source in the 1-12 keV band as seen by the EPIC MOS-2 camera is shown in Figure 1. Analysis does not reveal any gross energy dependence associated with the variability (section 3.2), which allows us to co-add the data from the whole observation to study the X-ray spectral shape.

\subsection{RGS data}

The summed RGS data are shown in Figure 2. The spectrum is characterised by a number of strong broad spectral features, peaking at approximately $19 \AA, 25 \AA$, and $34 \AA$, cut into by numerous narrow absorption lines. Overall the spectrum is similar in appearance to that reported by BR01, but the statistical quality is significantly higher. This is because the present observation was approximately twice as long, and because the average flux level over the observation was $50 \%$ brighter than it was in the data of BR01. A segment of the data is plotted on a larger scale in Figure 3, and compared with the spectrum derived from the BR01 observation. In this wavelength interval, the median signal to noise ratio per channel is 19 in the present data, compared to a value of 9 for the BR01 data. The two spectra are very similar, apart from excess narrow emission in the BR01 data near 16.4 and $17.5 \AA$ which may be due to higher order iron lines. The narrow absorption lines are unresolved, and have a width that is consistent with the instrumental resolution.

We try to fit the spectrum with two different schemes. In the first the broad spectral features are modelled as edges imprinted on a continuous underlying spectrum by a combination of neutral atoms in dust and a warm absorbing medium, both in the line of sight to the X-ray source. In the second the features are modelled as broad emission lines from a relativistic accretion disk, coupled with a foreground warm absorber. Both models include Galactic absorption with a column density of $1.77 \times 10^{20} \mathrm{H}$ atoms $\mathrm{cm}^{-2}$. We use crosssections for the Galactic O I edge computed by McLaughlin \& Kirby (1998) and include the Galactic O I 1s-2p resonance line and Fe I L 2p-3d resonances. We used data from Behar, Sako \& Kahn (2001) for the Fe I L cross sections. 


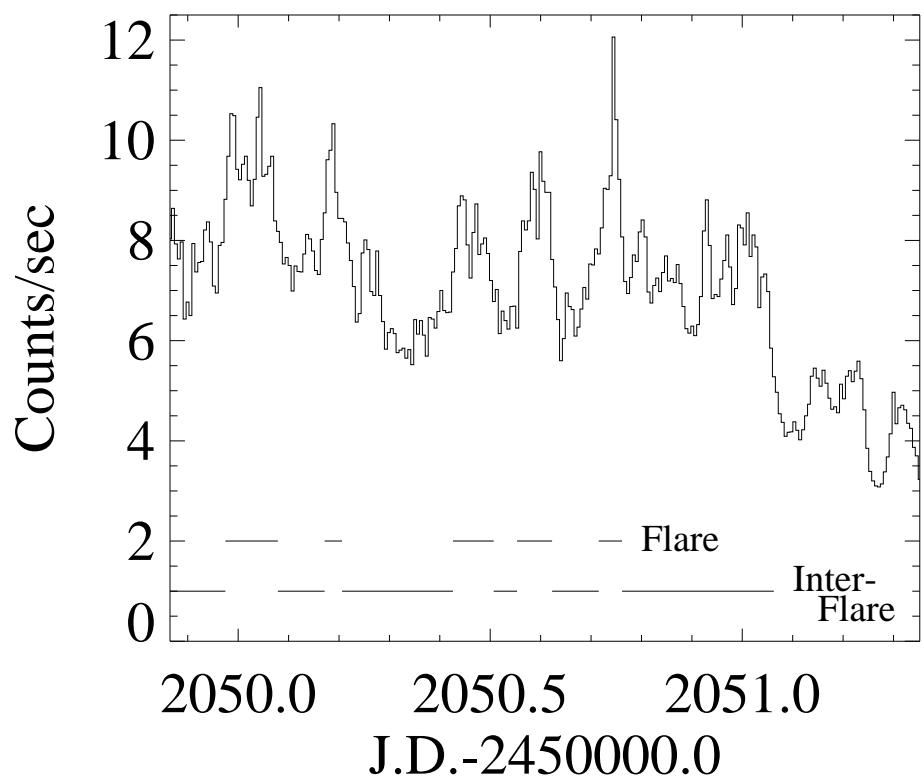

Fig. 1. - Light curve of Mrk 766 in the $1-12 \mathrm{keV}$ band taken with the EPIC MOS-2 camera during the current observation. The data are collected in 500s bins. Date intervals used for later spectral analysis are indicated. These are categorised as flaring ('Flare') or between flares ('Inter'). 


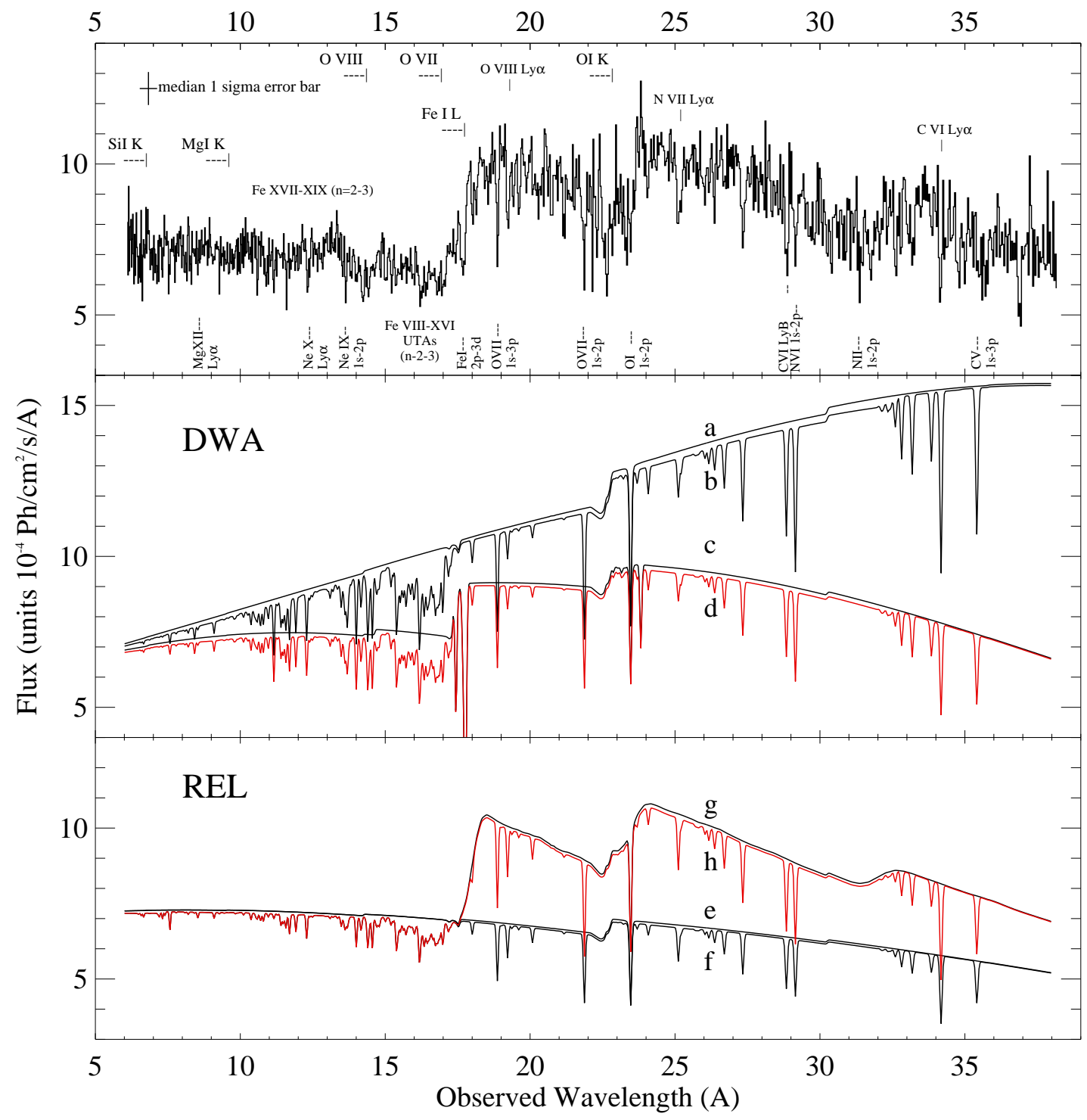

Fig. 2.- Upper panel: Combined RGS-1 and RGS-2 data (first and second order) on Mrk 766 summed over the 130ks observation and divided by the wavelength-dependent effective area of the instrument. The wavelength of various features that might be expected in the spectrum are marked. In the lower panels we show the components of the DWA and REL models that best fit the data. In each case, the red curve (curves $(d)$ and $(h)$ respectively for the DWA and REL case) is the combined best-fit spectrum. Each curve is modified by Galactic absorption. In the DWA fit, curve (a) is the combination of two power laws with photon slopes of 2.1 and 3.7 respectively, curve (b) includes the power law continuum and the warm absorber (dust omitted), while curve (c) shows the power law continuum plus dust (warm absorber omitted). In the REL fit, curve (e) is the single power law continuum with photon slope 2.15 , curve $(f)$ shows the power law continuum together with the warm absorber (emission lines omitted) while curve $(g)$ shows the power law continuum plus the broad emission lines (warm absorber omitted). 

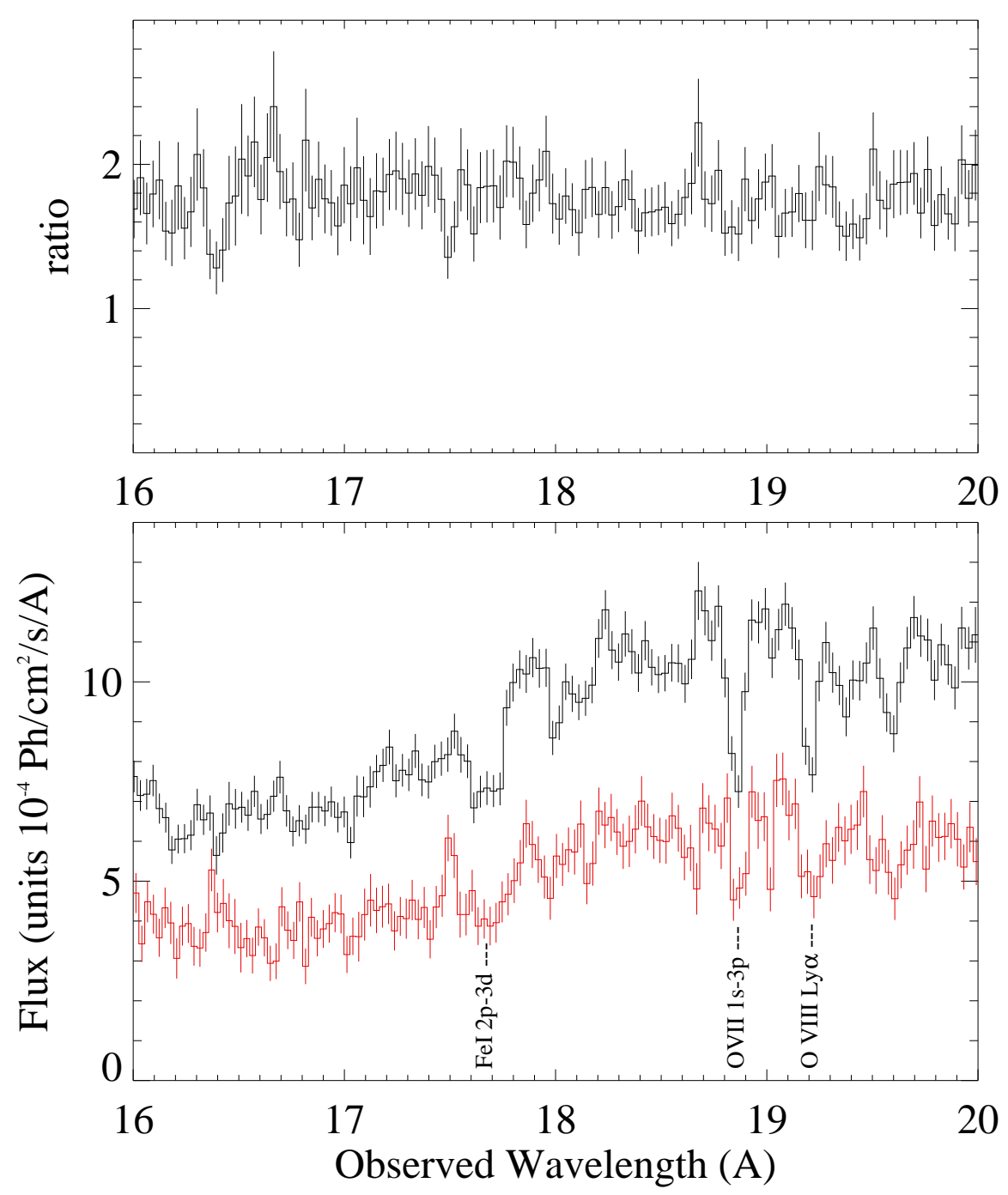

Fig. 3.- A section of the RGS spectrum from the present observation plotted on an expanded scale (black), compared to the spectrum from the Performance Verification phase obseration of BR01 (red), reduced with the same version of the analysis software. The ratio of the two spectra is shown in the upper plot. 


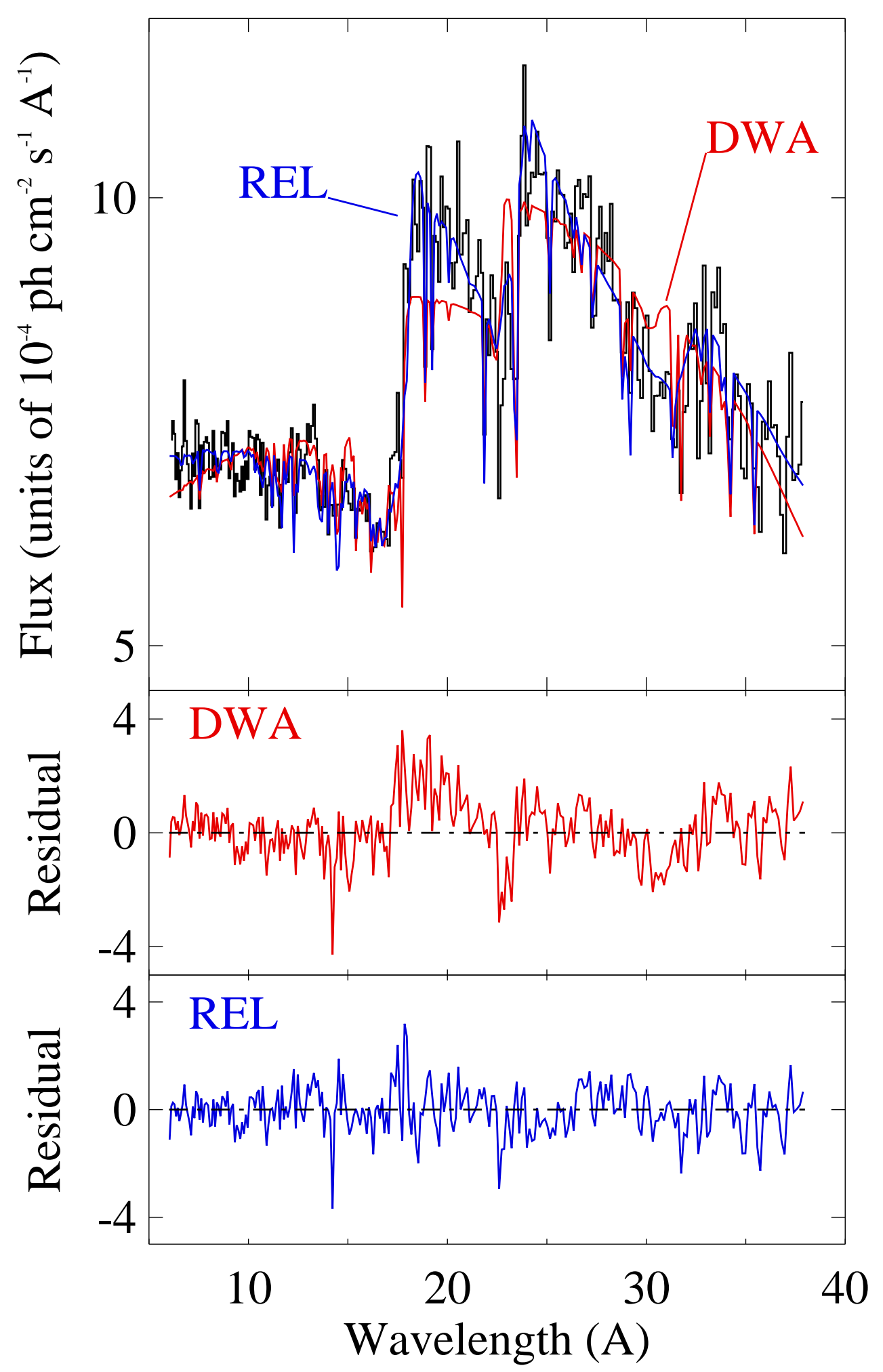

Fig. 4.- Comparison of the DWA (red) and REL (blue) model fits to the RGS data. For clarity of display, all data have been binned by a factor of four. 

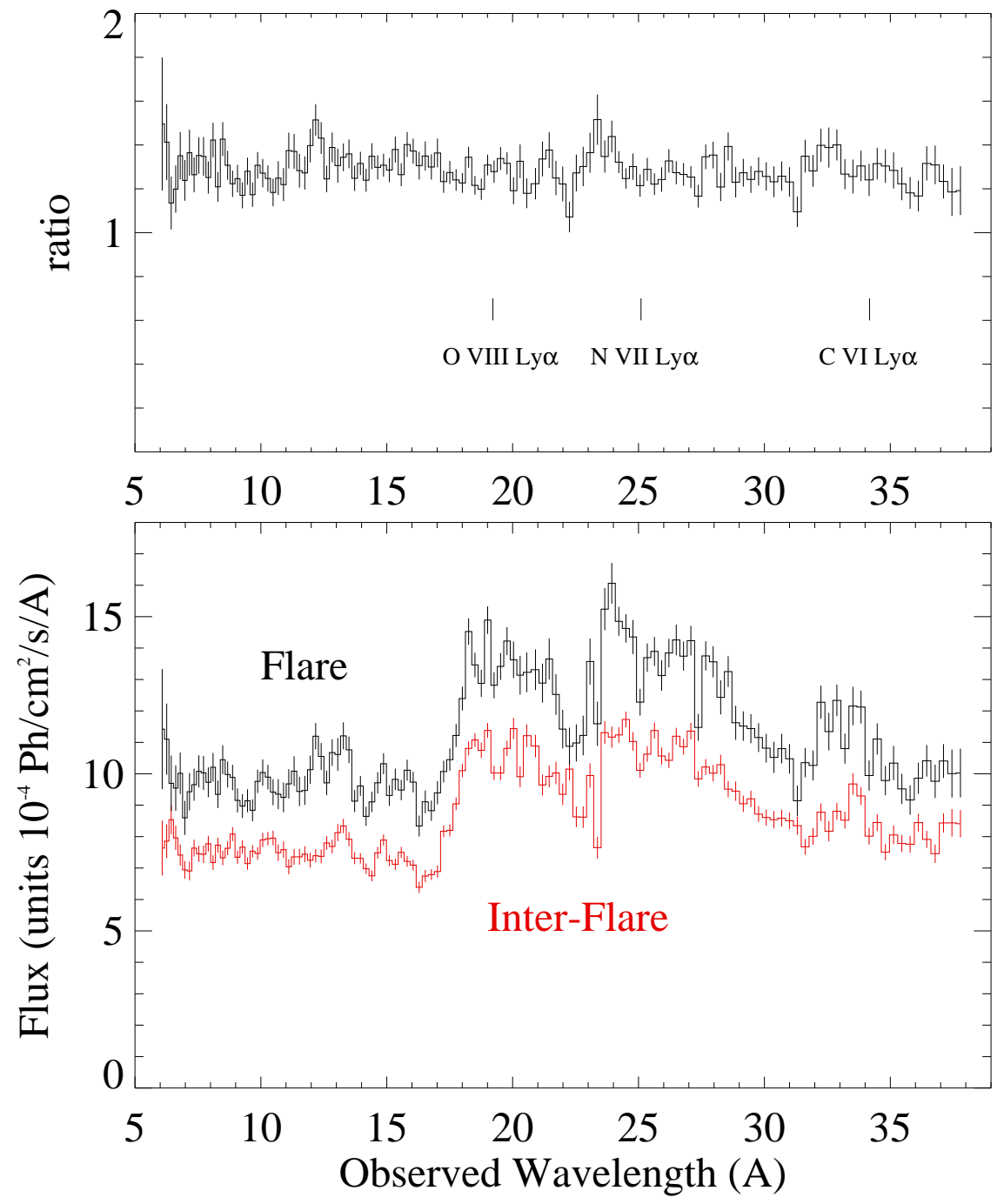

Fig. 5.- The RGS spectrum of Mrk 766 integrated over the peak of flaring intervals (see Figure 1) compared with the spectrum integrated over intervals between flares. The ratio of the two spectra is plotted in the upper panel. One sigma statistical errors are shown. The data have been binned by a factor of eight compared to Figure 2 to improve statistics. 

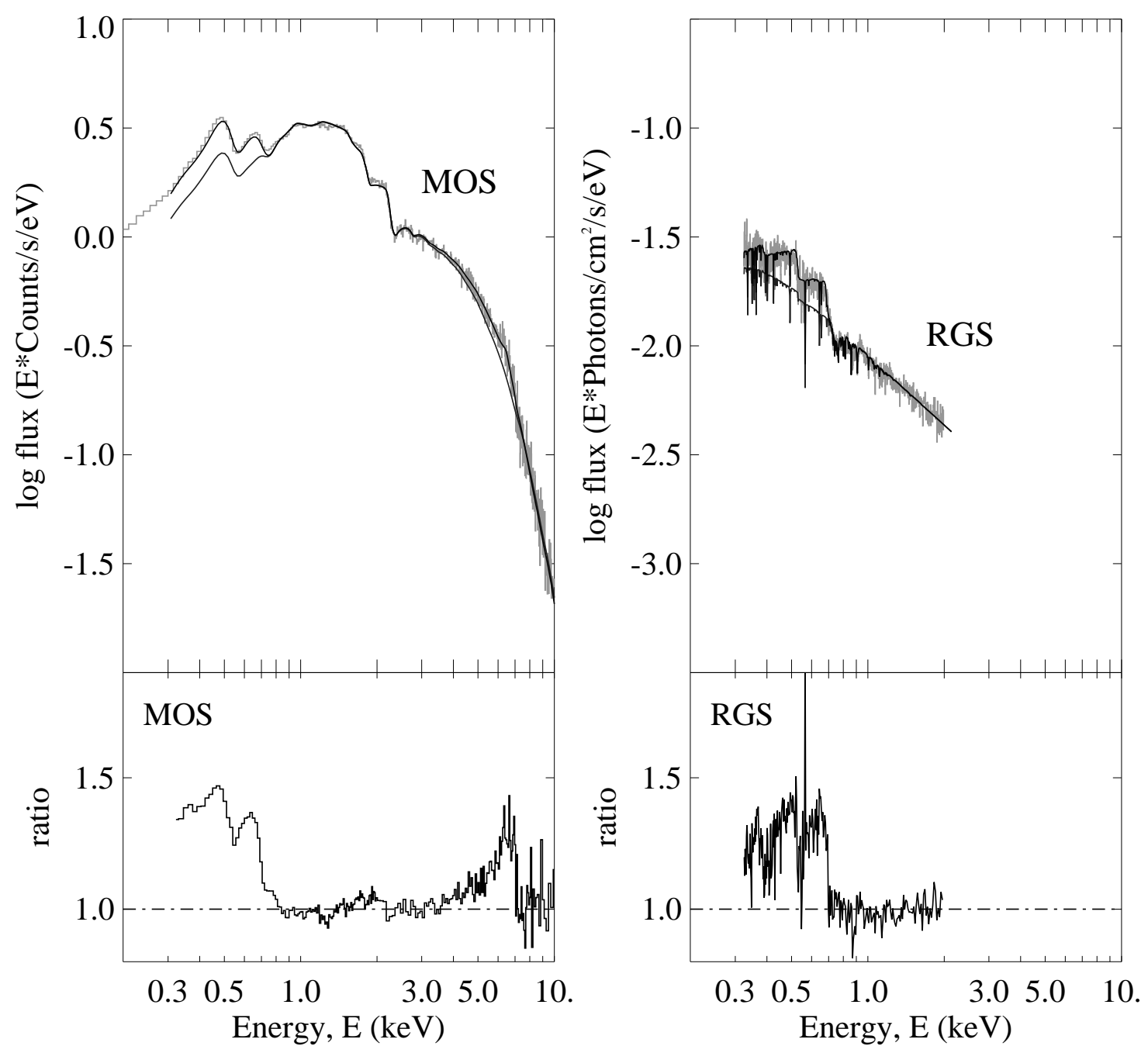

Fig. 6. - Left: EPIC MOS-2 count spectrum of Mrk 766 showing the best-fit REL model. Right:The RGS data from Figure 2 are also plotted, fitted to the same model. The model with the emission lines suppressed is included for both MOS and RGS to illustrate the contribution of the lines. The ratio of the data to the underlying spectrum with the lines removed is shown in the lower panels. 

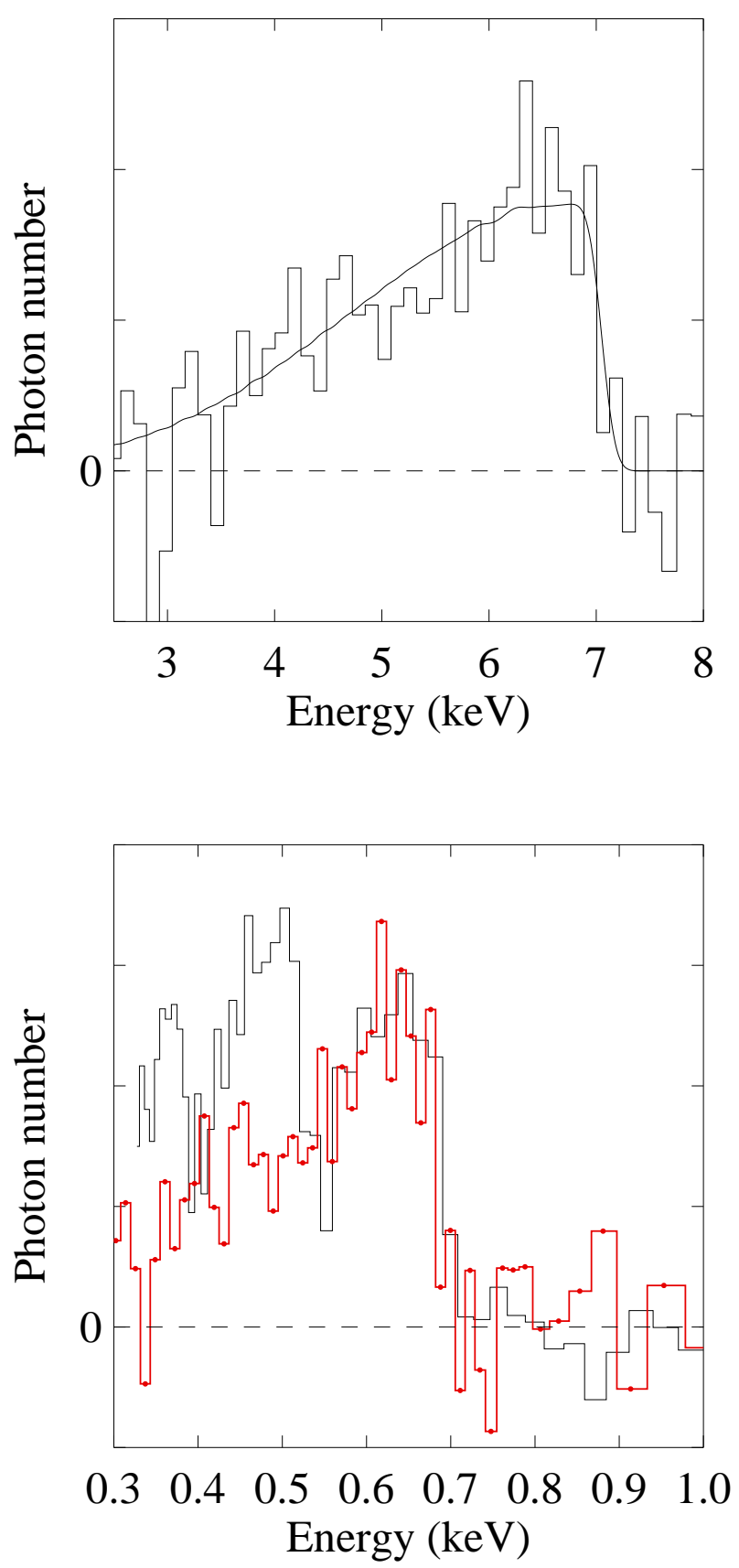

Fig. 7.- The upper panel shows the iron $\mathrm{K}_{\alpha}$ line profile of Mrk 766 derived from the EPIC MOS-2 data: the histogram shows the residuals from the best fit continuum illustrated in Figure 6. The continuum is derived from a model which includes a Laor line to describe the excess attributed to iron emission, and the best fit Laor profile is superimposed on the data as the continuous line. In the lower panel we show the RGS data on Mrk 766 with the best fit continuum subtracted. The red histogram shows the iron $\mathrm{K}_{\alpha}$ line data from MOS-2 scaled to the energy of the O VIII line. The data have been binned for clarity, and the relative line fluxes have been normalised to their peak values. 


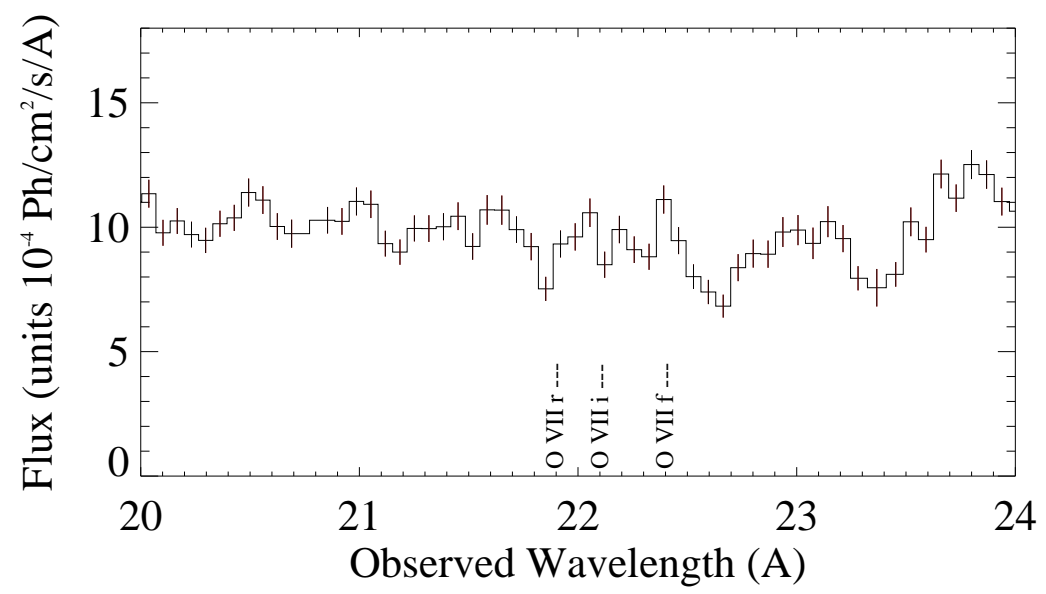

Fig. 8.- An expanded view of the RGS spectrum of Mrk 766 in the vicinity of the O VII triplet, summed over the whole observation. 


\subsubsection{Dusty Warm Absorber}

We have developed a Dusty Warm Absorber (DWA) model that we fit to the Mrk 766 RGS data. The model incorporates an ionized (warm) absorber component, which includes all abundant H-like and He-like ions (Table 1). For each ion we include K edges and resonance absorption lines from the ground state and L-shell absorption from ions Fe VII-XXIV. We model the warm absorber empirically, allowing the column density of each ion to vary so as to obtain a best fit. The Doppler b parameter $(=\sqrt{2} \sigma=0.6 F W H M)$ of the lines is fixed at an assumed $100 \mathrm{~km} / \mathrm{s}$. The narrow lines are not resolved by the RGS and we can place an upper limit on their directly measured width of $b=300 \mathrm{~km} / \mathrm{s}$. We can however constrain the $\mathrm{b}$ parameter by comparing the equivalent widths of different lines produced by the same ion. The formal best-fit, using the 18-38 $\AA$ range to avoid the blended iron lines at shorter wavelengths, is $b=95 \pm 30 \mathrm{~km} \mathrm{~s}^{-1}$. There is no evidence for a global velocity shift of the lines greater than $\pm 100 \mathrm{~km} / \mathrm{s}$ with respect the systemic velocity $(\mathrm{z}=0.012929)$.

We include neutral absorption in the model from the $\mathrm{K}, \mathrm{L}$ and $\mathrm{M}$ edges of $\mathrm{Fe} \mathrm{I}$ and O I, and other abundant elements that might be present including: C I, Mg I, Ne I, and Si I. Neutral absorption edges might arise in dust that is embedded in the warm absorber, as suggested by Reynolds et al. (1997; see also Komossa \& Bade 1998).

In modelling the $\mathrm{H}$-like and He-like resonance lines up to $\mathrm{n}=10$, we use atomic data from Verner et al. (1996a). Data for $n=11-30$ are extrapolated using the hydrogenic approximation. The Fe-L shell oscillator strengths and natural line widths have been computed by Behar et al. (2001). The lines are represented by Voigt profiles, convolved with the instrument broadening function. The edges are modelled using cross sections calculated by Verner et al. (1996b).

Below about $0.7 \mathrm{keV}$ Mrk 766 exhibits an excess above an extrapolation of the best-fit medium energy power-law (e.g. Page et al. 2001). We thus require a soft excess spectral component if we are to fit the overall spectrum with a DWA model, since the DWA itself can only remove flux. We tried a blackbody spectrum to represent this soft excess, but a power law was a much better fit so we have adopted this in what follows. Galactic absorption with a column density $N_{H}=1.77 \times 10^{20} \mathrm{~cm}^{-2}$ was included.

The column densities associated with the various warm absorber lines and dust features were fit along with the continuum parameters so as to minimise $\chi^{2}$. The individual components that make up the best fit DWA model are illustrated in the middle panel of Figure 2. The overall model is compared with the data directly in Figure 4 , and has a $\chi^{2} /$ d.o.f. $=2.2$ (1094 d.o.f.). The counterbalancing effects of the steep low energy excess and the absorption by neutral atoms inherent in the model are clear from Figure 2. The best fit slope of the 
Table 1. Ionized Warm Absorber

\begin{tabular}{lllllllll}
\hline \hline Ion & DWA N & REL N & Ion & DWA N & REL N $_{i}$ & Ion & DWA N & REL N $_{i}$ \\
\hline C V & 3.4 & 2.2 & Fe VIII & 0.23 & 0.10 & Fe XVII & 0.79 & 0.39 \\
C VI & 3.4 & 3.7 & Fe IX & 0.45 & 0.24 & Fe XVIII & 0.94 & 0.48 \\
N VI & 0.58 & 0.42 & Fe X & 0.72 & 0.43 & Fe XIX & 0.38 & 0.11 \\
N VII & 0.72 & 1.4 & Fe XI & 0.19 & 0.12 & Fe XX & 0 & 0 \\
O VII & 2.2 & 1.8 & Fe XII & 0.62 & 0.37 & Fe XXI & 0 & 0 \\
O VIII & 0.56 & 1.2 & Fe XIII & 0.12 & 0.10 & Fe XXII & 0.57 & 0.30 \\
Ne IX & 1.1 & 0.57 & Fe XIV & 0.36 & 0.10 & Fe XXIII & 2.0 & 1.5 \\
Ne X & 0.46 & 0.25 & Fe XV & 0.33 & 0.16 & Fe XXIV & 0.86 & 0.46 \\
Fe VII & 0 & 0 & Fe XVI & 0.29 & 0.16 & & & \\
\hline
\end{tabular}

Note. - units $10^{16} \mathrm{~cm}^{-2}$

Table 2. DWA Neutral Absorber

\begin{tabular}{lc}
\hline \hline Ion & $\begin{array}{r}\text { Column Density (units } \\
\left.10^{16} \mathrm{~cm}^{-2}\right)\end{array}$ \\
\hline C I & $97.7_{-0.4}^{+3.3}$ \\
O I & $2.4_{-1.7}^{+1.5}$ \\
Mg I & $0.0_{-0.2}^{+4.2}$ \\
Si I & $2.3_{-0.3}^{+2.1}$ \\
Fe I & $16.5_{-0.2}^{+1.3}$ \\
\hline
\end{tabular}

Note. - errors are $90 \%$ confidence. 
underlying hard power law is $\Gamma=2.1$, while $\Gamma=3.7$ for the soft excess power law. The flux at $1 \mathrm{keV}$ is $5.4 \times 10^{-4}$ photons $/ \mathrm{cm}^{2} / \mathrm{s} / \AA$ in the $\Gamma=2.1$ power law and $3.3 \times 10^{-6}$ photons $/ \mathrm{cm}^{2} / \mathrm{s} /$ Ain the $\Gamma=3.7$ power law.

Overall, the broad spectral features in the DWA model are too shallow compared to the data. The depth of the edges could be increased if the flux in the underlying power law were higher, but the overall slope of the resulting spectrum would not match the data. A combination of the Fe I edge and the $n=2-3$ lines near $16 \AA$ (Behar et al. 2001) causes the flux to rise between $17 \AA$ and $19 \AA$, but not as much as observed in the data. The rise in X-ray flux between $\sim 22 \AA$ and $\sim 24 \AA$ is not well reproduced by a combination of the Galactic neutral oxygen $\mathrm{K}$ edge and $\mathrm{O} \mathrm{I}$ absorption in the Seyfert, and neither does the model reproduce the rise in flux between $\sim 31 \AA$ and $\sim 33 \AA$.

The best fit neutral column densities are summarised in Table 2. The species with the largest neutral column densities are carbon and iron. However, the neutral carbon edge is not within the RGS wavelength range.

Given the size of the neutral iron edge that is fitted, we would expect to see a much stronger Fe I absorption line at $\sim 17.7 \AA$ in the observed frame (see Figure 2 ). To measure the observed strength of this line as accurately as possible, we have modelled the continuum in the $16 \AA$ to $22 \AA$ region with a fifth order polynomial, together with absorption lines due to O VII, $\mathrm{O}$ VIII and Fe I. Use of a local polynomial continuum minimises systematic uncertainties due to inadequacies in the global model. The Fe I line is blended with O VII 1s-5p, and we measure a combined equivalent width of $38 \pm 9 \mathrm{~m} \AA$. Ascribing half of this, roughly $20 \mathrm{~m} \AA$, to Fe I, we derive an Fe I column of $2.3 \pm 0.4 \times 10^{16} \mathrm{~cm}^{-2}$ for a Doppler b width of $100 \mathrm{~km} / \mathrm{s}$. The line is on the linear part of the curve of growth, and it is also an unresolved transition array (UTA; Behar et al. 2001) with an effective width of $600 \mathrm{~km} \mathrm{~s}^{-1}$. Thus the derived column density is insensitive to uncertainties in $\mathrm{b}$, and only increases to $2.9 \pm 0.5 \times 10^{16} \mathrm{~cm}^{-2}$ if we assume $\mathrm{b}=50 \mathrm{~km} / \mathrm{s}$. This is a factor of 5-7 times less than the column density required by the fits to the neutral iron edge in the DWA model (Table 2). Conversely, based on the measured strength of the 17.7A line, the neutral iron edge is expected to be weak and can not explain the large $17 \mathrm{~A}$ continuum break.

\subsubsection{Relativistic Emission Lines}

The second model seeks to fit the soft X-ray spectral features with emission lines from an ionized, relativistic accretion disk (BR01, Laor 1991). Relativistic broadening has been invoked previously to explain the profile of the iron emission line near $6 \mathrm{keV}$ observed first 
from MCG-6-30-15 using ASCA (Tanaka et al. 1995; see also Lubiński \& Zdziarski 2001 and references therein). Following BR01 we fit the RGS spectrum with emission lines due to H-like Ly $\alpha$ lines of O VIII, N VII and C VI. The model includes a warm absorber component with identical atomic data to that used in the DWA fit. As in the DWA model, we allow the column density of each warm absorber ion to vary to get the best fit, and assume $b=100$ $\mathrm{km} / \mathrm{s}$. Unlike the DWA model, only a single power law continuum is required in the REL fit.

The contribution of the individual components that make up the Relativistic Emission Line (REL) model is illustrated in the lower panel of Figure 2. Overall, the best fit REL model (Figure 4 ) has a $\chi^{2} /$ d.o.f.=1.7, significantly better than the DWA fit. The REL model is more successful in reproducing the overall shape of the observed spectrum, and consequently the systematic deviations of the residuals plotted in Figure 4 are less marked than those of the DWA fit. The characteristic sawtooth rises in flux at $\sim 18 \AA, \sim 23 \AA$ and $\sim 32 \AA$ are modelled by the overlapping asymmetric profiles of O VIII, N VII and C VI emission lines. The line energies are consistent with the Ly $\alpha$ transitions of these ions as reported by BR01.

While visibly a better fit, the formal value of $\chi^{2}$ for the REL model is still high. There is a significant contribution to the reduced $\chi^{2}$ of both the REL and DWA fits from inner shell resonances of low ionization species (NI-V, OI-VI, etc), which we have not yet included in either model. These do not affect the relative comparison between them however.

We have assessed the contribution of other potential disk emission lines to the observed spectrum, particularly O VII which is predicted in the accretion disk models of Ballantyne, Ross \& Fabian (2002). Assuming the same profile as the O VIII line, we can place a $3 \sigma$ upper limit on the flux of O VII relative to O VIII of $\sim 0.11$.

The best fit continuum slope for the REL model has $\Gamma=2.15$, with a flux at $1 \mathrm{keV}$ of $6.8 \times 10^{-4}$ photons $/ \mathrm{cm}^{2} / \mathrm{s} / \AA$. The line profiles are consistent with a common set of accretion disk parameters as listed in Table 3 , and are indicative of emission from just outside the last stable orbit about the black hole $\left(\mathrm{R}_{i}=1.24 \mathrm{R}_{\mathrm{G}}\right)$. The emissivity index of the accretion disk is steep $(\sim 3.7)$ and is similar for the three ions. This means that $86 \%$ of the disk emission occurs within $6 \mathrm{R}_{\mathrm{G}}$ of the black hole. The lines are too broad to be explained by emission outside the last stable orbit of a non-rotating (Schwarzschild) black hole. The blue shoulders of the model lines have a peak-to-base width of about $\Delta \lambda / \lambda=0.05$ which is equivalent to $\sim 33 \mathrm{eV}(0.9 \AA)$ for O VIII. This corresponds to an accretion disk inclination of $35^{\circ}$. 
Table 3. Relativistic Emission Lines

\begin{tabular}{lcccl}
\hline \hline \multicolumn{1}{c}{ Parameter } & C VI & N VII & O VIII & Fe K \\
\hline E(keV: C,N,O fixed) & 0.3675 & 0.5003 & 0.6536 & $6.73 \pm 0.07$ \\
$\mathrm{R}_{\text {in }}\left(\mathrm{R}_{G}\right)$ & $1.24_{-i n f}^{+0.45}$ & $1.24_{-i n f}^{+0.30}$ & $1.24_{-i n f}^{+0.25}$ & $1.32_{-0.09}^{+0.80}$ \\
Emissivity Index & $3.79_{-0.06}^{+0.28}$ & $3.73_{-0.04}^{+0.07}$ & $3.51_{-0.02}^{+0.04}$ & $3.53_{-0.19}^{+0.19}$ \\
Flux $\left(10^{-3} \mathrm{ph} \mathrm{s}^{-1} \mathrm{~cm}^{-2}\right)$ & $2.99_{-0.67}^{+0.12}$ & $3.65_{-0.23}^{+0.06}$ & $3.71_{-0.19}^{+0.03}$ & $0.12 \pm 0.1$ \\
EW $(\mathrm{eV})$ & $42_{-9}^{+2}$ & $96_{-6}^{+2}$ & $169_{-9}^{+1}$ & $684 \pm 55$ \\
\hline
\end{tabular}

Note. - fixed parameters: redshift $=0.012929$, disk inclination $35^{\circ}$, disk outer radius $=400 \mathrm{R}_{G}$. Errors are $90 \%$ confidence.

Table 4. Warm absorber: Equivalent hydrogen column densities

\begin{tabular}{lcc}
\hline \hline Element (Ion) & $\mathrm{N}_{\mathrm{H}}(\mathrm{DWA})$ & $\mathrm{N}_{\mathrm{H}}(\mathrm{Rel})$ \\
& & \\
\hline $\mathrm{C}(\mathrm{V}-\mathrm{VI})$ & 1.9 & 1.6 \\
$\mathrm{~N}(\mathrm{VI}-\mathrm{VII})$ & 1.2 & 1.6 \\
$\mathrm{O}($ VII-VIII) & 0.33 & 0.35 \\
$\mathrm{Ne}(\mathrm{IX}-\mathrm{X})$ & 1.3 & 0.66 \\
$\mathrm{Fe}($ VII-XXIV) & 18.9 & 10.7 \\
\hline
\end{tabular}

Note. - units are $10^{20} \mathrm{~cm}^{-2}$. 


\subsection{Spectral Variability}

To search for variability of the RGS spectral features with source intensity, we have divided the data according to whether they are taken around a peak of the 1-2 hour timescale flares in the light curve, or in the interflare period. The time intervals used are marked in Figure 1. We have not used data from the low state that occurs towards the end of the observation in the variability analysis. This is because the total number of source counts is significantly less in this time period. Moreover there is an increase in the background associated with the end of the spacecraft orbit that further degrades the statistical precision of the data during the low-flux interval. The individual 'flare' and 'interflare' spectra are plotted in Figure 5, where we have binned the data in wavelength to increase signal to noise. The ratio of the two spectra are shown in the upper plot of the Figure.

Overall, the high- and low-flux spectra have a similar shape and show the same spectral features. The overall ratio is relatively flat, supporting our earlier assertions that the flux variability in Mrk 766 is not associated with a gross change in spectral shape. The $\chi^{2}$ for a fit of the ratio to a horizontal line is 151 for 123 degrees of freedom. Nevertherless there are significant deviations from the mean at about the $\pm 15 \%$ level. The most prominent are a peak in the ratio at about $12 \AA$, and a dip at about $22 \AA$ accompanied by an excess with similar significance to longer wavelengths. In each feature the maximum deviation of individual points from the mean is only slightly more than 3 sigma, but the deviations are systematic over a number of neighbouring points. The excess ratio near $12 \AA$ may be due to a complex of Fe XXII and Ne X emission. The centroid of the dip at $22 \AA(22.2 \AA)$ is close to the wavelength of low-level O VII forbidden line emission seen in the spectrum $(22.38 \AA$ in the observed frame; see section 3.3) but the width and flux of the forbidden line are insufficient to account for the feature we see, and its centroid is significantly different. The $22 \AA$ feature coincides with the blue wing of the putative broad N VII line, and it may be that we are seeing changes in either the blue wing of the N VII line or the red wing of the O VIII line during flares. Interestingly, there may also be a lower significance feature shortward of the

C VI Ly $\alpha$ rest wavelength at about $31 \AA$, but the reality of this feature is less certain. There is no significant change in ratio associated with the blue wing of the O VIII line.

\subsection{Warm Absorber}

The presence of a warm absorber is betrayed by the numerous narrow absorption lines of ionized species in the RGS spectrum of Mrk 766. As noted previously, our approach is to model these lines empirically as we seek to understand the nature of the underlying continuum. In Table 1 we list the column density of individual ions for both the DWA and 
REL models. These column densities are naturally only meaningful if the underlying model accurately reproduces the continuum in the vicinity of the relevant line, so generally the values for the REL model are most representative since this is a better approximation to the observed continuum. We also stress that the column densities will be sensitive to the Doppler line width assumed (see section 3.1.1). Furthermore the absorption lines are likely to be filled in to some extent by emission from the extended warm absorber gas.

There is indeed weak O VII forbidden emission in Mrk 766 near $22 \AA$ (Figure 8) and this can be used to roughly estimate the covering fraction of the warm absorber by comparing it to the measured flux absorbed by the O VII 1s-2p resonance line. We measure a flux in the forbidden emission line ( $\mathrm{f}$ in Figure 8) of $1.5 \pm 0.8 \times 10^{-5} \mathrm{ph} \mathrm{cm}^{-2} \mathrm{~s}^{-1}$, and absorbed flux in the O VII $1 \mathrm{~s}-2 \mathrm{p}$ resonance $(\mathrm{r})$ line of $1.9 \pm 0.7 \times 10^{-5} \mathrm{ph} \mathrm{cm}^{-2} \mathrm{~s}^{-1}$. The ratio of the forbidden line emission flux to the corresponding emission in the $2 \mathrm{p}-1 \mathrm{~s}$ line can be estimated based on measurements of NGC 1068, where Kinkhabwala et al. (2002) find a ratio of 2.1. Thus, assuming similar densities for the warm absorbers in Mrk 766 and NGC 1068, we expect emission from O VII $2 \mathrm{p}-1 \mathrm{~s}$ at a level of $0.71 \times 10^{-5} \mathrm{ph} \mathrm{cm}^{-2} \mathrm{~s}^{-1}$. This means that $30 \%$ of the O VII 1s-2p absorption line is expected to be filled by emission and the corrected absorption line flux is therefore $\sim 2.6 \pm 0.7 \times 10^{-5} \mathrm{ph} \mathrm{cm}^{-2} \mathrm{~s}^{-1}$. The ratio of resonantly scattered to total flux in the $1 \mathrm{~s}-2 \mathrm{p}$ emission line in NGC 1068 is found to be 0.44 . If we assume that this is also representative of Mrk 766, we derive an estimate of about $12 \%$ for the covering factor of the warm absorber in the latter. This number should be treated with caution, since in practice the scaling from NGC 1068 is likely to be sensitive to differences in the mean column density and clumpiness of the warm absorber in the two sources. Nevertheless this exercise demonstrates that the implied warm absorber covering factor in Mrk 766 is at least reasonable.

In Table 4 we list the equivalent hydrogen column densities of the principal line-forming elements in the warm absorber, summed over the ionic species measured, and assuming cosmic abundance. As before these values assume $b=100 \mathrm{~km} / \mathrm{s}$. The implied column of oxygen in the warm absorber is a factor of four lower than either carbon or nitrogen while the column density derived from the iron lines is an order of magnitude higher than from the other measured elements. We note in passing that the relative strength of the broad $\mathrm{N}$ VII and O VIII emission lines fitted in the REL model also requires that the nitrogen abundance relative to oxygen is higher than the solar value by a similar factor, as noted by BR01. The iron column in the warm absorber is summed over a much larger range of ionisation states than the other elements and it is possible that carbon, nitrogen and oxygen exist largely in ionisation states that we have not measured. Strictly speaking, a physical ionisation model of the warm absorber gas is required to interpret these column densities convincingly in terms of elemental abundances. However, the observed overabundance of 
iron, by an order of magnitude, is rather large to be explained away in this manner. We note that a similar overabundance of iron has been measured in NGC 3783 (Blustin et al. 2002) and IRAS 13349+2438 (Sako et al. 2001).

We should recognise however that these values are likely to be sensitive to uncertainties in the Doppler width, b, of the lines, and that the sensitivity will be different for different ions. For example, when we use $b=65 \mathrm{~km} / \mathrm{s}$ the column density of carbon is about three times higher, with lesser increases for nitrogen and oxygen. The column density of iron is less sensitive to $b$, however, since many of the iron features are UTAs (see section 3.1.1). Thus a decrease in b would serve to decrease the abundance discrepancy between iron and the lighter elements.

\subsection{MOS and RGS data}

The EPIC MOS-2 data are shown in Figure 6. The sawtooth shaped spectral features that are shown to advantage by the RGS data are also clearly present in the MOS. We have fitted the data with the REL model described above, simultaneously with the RGS data, adding a fourth relativistically broadened line at $6.7 \mathrm{keV}$ to represent iron emission. The best fit power law continuum parameters are indistinguishable from those that were derived from the RGS data alone. While this model is a reasonable representation of the data over most of the energy range, a simple power-law does underpredict the flux above the iron line by an amount that rises to $30 \%$ at $10 \mathrm{keV}$. Because the response of the instrument falls off towards high energies, this excess has relatively little statistical weight in the overall fit however. We can model the excess with a power-law that has a break above $5.6 \mathrm{keV}$, hardening by $\Delta \Gamma=0.4$ to accommodate the high energy excess. This improves the reduced $\chi^{2}$ from 2.2 to 2.1 per d.o.f for 1624 d.o.f. The broken power-law model fit is illustrated in Figure 6. It is likely that the high energy excess is due to reflection from a disk. Indeed we can represent the spectrum equally well (i.e. identical $\chi^{2}$ ) with a model (developed by Piotr Zycki) that includes reflection from a constant density X-ray illuminated atmosphere. It computes the Compton-reflected continuum (cf. Magdziarz \& Zdziarski, 1995) and the Fe K $\alpha$ line (cf. Zycki \& Czerny, 1994) and adds relativistic smearing around a Schwarzschild black hole.

The reduced $\chi^{2}$ of the best-fit REL model is still formally poor, at 2.1 per d.o.f. However, as evidenced by Figure 6, the systematic deviations of the data from the model are small, and the poor reduced $\chi^{2}$ is largely due to unmodelled low-level spectral complexity, particularly at the lowest energies. As with the RGS data on their own, the DWA model (together with a relativistic $\mathrm{Fe} \mathrm{K} \alpha$ line and high energy excess) is a significantly poorer fit to the 
combined MOS and RGS data than the REL model, yielding $\chi^{2} /$ d.o.f=3.0. Significantly, even over the extended energy range covered by the MOS, the REL model still requires only a single underlying $\Gamma=2.15$ power law continuum to fit the data, provided we include a disk reflection component, or its equivalent, to account for the high energy excess.

In Figure 7 we plot the residuals of the MOS-2 data in the $3-8 \mathrm{keV}$ range with respect to the best fitting broken power-law continuum. We use this simple continuum rather than the full reflection spectrum model to illustrate the actual shape of the observed data in the vicinity of the iron line without introducing model-dependent line-like features. We also show the REL model profile (Laor 1991) used to fit the iron line in the broken power-law model, the parameters of which are listed in Table 3. The data support the notion of a strongly asymmetric line whose red wing extends down to about $3 \mathrm{keV}$. Its energy is indicative of highly ionized iron, and the profile is consistent with the same set of disk parameters used for the low-energy lines (Table 3). The detection of the red wing in the iron line confirms the report by Page et al. (2001). In the lower panel of Figure 7 we have scaled the MOS-2 iron line profile to the energy of the O VIII line and superimpose this on the RGS data. There is good agreement between the MOS and RGS profiles, quantified by the conjunction of the model line parameters and lending credence to the idea that the RGS spectral features are caused by relativistic emission lines.

\section{Discussion}

The long observation of Mrk 766 reported in this paper, which sampled the source at a higher mean flux level than previously, provides the opportunity to test the rival models that have been put forward to explain the low-energy spectral features first reported by BR01. We have sufficient signal-to-noise to constrain the variability of these features as a function of source flux. We have also used the EPIC instrument on XMM-Newton to confirm the presence of a broad, asymmetric iron line feature at $6.7 \mathrm{keV}$, and use the combination of the RGS and EPIC instruments on XMM-Newton to compare the iron line profile with the low-energy spectrum.

A point of debate in the literature has been the discontinuity in the spectrum of Mrk 766 at about $18 \AA$. A similar feature has also been seen in MCG-6-30-15 by BR01 and Lee et al. (2001). Conceivably it is an absorption edge. Its wavelength, however, is inconsistent with the OVII edge expected from a warm absorber unless the material producing it is redshifted by an implausible amount $(\sim 16,000 \mathrm{~km} / \mathrm{s})$. Thus Lee et al. (2001) propose that the feature in MCG-6-30-15 is due to the L-edge of neutral iron, which matches the observed wavelength more closely than the OVII edge. When interpreted in this way, the depth of the feature in 
MCG-6-30-15 implies an equivalent hydrogen column of $N_{H}=4 \times 10^{21} \mathrm{~cm}^{-2}$ assuming solar abundances. This cannot be a column of neutral gas, otherwise the soft X-ray spectrum would be highly absorbed by CI and OI edges, which is not seen. Besides, the presence of numerous narrow absorption lines from ionized species is indicative of a warm absorber. This leads Lee et al. to conclude that the neutral fraction of the absorber, particularly iron, is bound up in dust that is immersed in the ionized medium. Such dusty warm absorber (DWA) models have previously been invoked to explain the apparent discrepancy between the large reddening of certain AGN deduced from their optical-UV spectrum, and the lack of corresponding absorption of the X-ray spectrum (e.g. Reynolds et al. 1997; Walter \& Fink 1993).

We have developed a DWA model and have fitted it to our RGS data on Mrk 766. The model struggles to reproduce the overall shape of the RGS spectrum. If we nevertheless interpret the sharp discontinuity in flux near $18 \AA$ as being due to neutral iron, the column density implied is $1.7 \times 10^{17} \mathrm{~cm}^{-2}$. This is of the right order to be consistent with the estimated reddening of Mrk $766(\mathrm{E}(\mathrm{B}-\mathrm{V})=0.4$; Walter \& Fink 1993) for reasonable abundances. Given the required depth of the Fe I L edge we would, though, expect to see much stronger Fe I L resonance lines than are observed. Furthermore, there is no compelling evidence for K edges of other neutral atoms such as O I, Mg I \& Si I at the redshift of Mrk 766, and the composition of the putative dust grains is therefore unphysical. The DWA fit also requires a significant column of neutral carbon, forced by the slope of the long-wavelength spectrum in the RGS data. The carbon edge itself is not within the RGS range, but the MOS data extend to these energies and beyond. Even accounting for uncertainties in the MOS response matrix at these energies, we find no evidence for the expected increase in flux below $0.2 \mathrm{keV}$, which should amount to a factor of two if the edge is present at the level implied by the fits to the RGS data.

In contrast, the relativistic emission line (REL) model is more successful in reproducing the Mrk 766 RGS 0.3-2 keV data. The broad spectral features are consistent with Ly $\alpha$ emission lines of O VIII, N VII and C VI at the rest wavelength of the galaxy, superimposed on an underlying power-law. An extension of this model including a fourth line to represent emission due to iron at $6.7 \mathrm{keV}$ and reflection from a disk, also fits the $0.2-10 \mathrm{keV}$ EPIC MOS data on Mrk 766. The profiles of the low energy emission lines in Mrk 766 are consistent with that measured for the iron emission at $6.7 \mathrm{keV}$. The REL model requires only a single underlying power law continuum (with the excess above $7 \mathrm{keV}$ being accounted for by reflection), unlike the DWA model where a steep low-energy excess component below about $0.7 \mathrm{keV}$ is also needed.

Thus, adding dust to a warm absorber is not a convincing description of the Mrk 766 
X-ray spectrum while the REL model, suggested for Mrk 766 originally by BR01 to explain the earlier, shorter XMM-Newton observation, has become more compelling when confronted with the higher signal to noise data reported here. In many ways Mrk 766 is a more clear-cut example of the phenomenon than MCG-6-30-15 because the spectrum is less affected by the warm absorber.

The underlying physics that might produce such a (REL) spectrum is still controversial. Ballantyne \& Fabian (2001) fit a model to the ASCA spectrum of MCG-6-30-15 that involves reflection of hard X-rays from ionized layers on the surface of an accretion disk. They optimise the model parameters to best fit the iron $\mathrm{K}$ line, but this falls short of reproducing the observed equivalent width of the low-energy lines by more than an order of magnitude. For example, their model predicts an equivalent width of only $6 \mathrm{eV}$ for the oxygen line assuming solar abundance. In the Ballantyne \& Fabian model, however, the iron $\mathrm{K}$ line is a mix of emission from an ionized inner disk, and a sharper neutral component from further out. It is not clear how well the ionisation state of the inner disk region is being constrained in the model.

In more recent work, Ballantyne, Ross \& Fabian (2002) relax the requirement to fit the iron line and thus widen the scope of parameter space explored. In this case there are regimes in which their model does predict equivalent widths for the low energy lines comparable to those that we measure. Sako et al. (2002) also describe a thermal/ionization structure for the accretion disk surface layers in which Ly- $\alpha$ recombination lines of appropriate equivalent width could plausibly be produced. The lines will be intrinsically broadened by Compton scattering in the hot disk atmosphere. However, the widths that Ballantyne et al. predict for the blue wing of the lines (a few tens of eV) are comparable to those that we measure for the underlying emission lines in our Mrk 766 data, when the effects of absorption line and edge features in the warm absorber are taken into account. The Ballantyne et al. models predict additional weaker line features due to O VII and N VI that are not required by our fits. However, the upper limit that we can set on an O VII line in our data, measured with respect to the observed flux of O VIII, is only marginally inconsistent with the predictions of the Ballantyne et al. model for an ionizing parameter $\left(L / n r^{2}\right)$ of $250 \mathrm{erg} \mathrm{cm} \mathrm{s}^{-1}$ and Solar oxygen abundance. Sako et al. (2002) in any case use a different ionisation structure for the hot layer, in which these lines are supressed. Sako et al. also argue that iron L-lines above the O VIII K edge at $14.22 \AA$, predicted by Ballantyne et al., will be absorbed and the energy re-radiated at $\mathrm{O}$ VIII Ly- $\alpha$.

We have searched for evidence of variability in the low-energy spectral features of Mrk 766 in response to the 1-2 hour timescale flares that are seen in the light curve. There are indications of a systematic change in the high-to-low flux ratio at the $\pm 15 \%$ level par- 
ticularly in the region between about $22 \AA$ and $24 \AA$. This signature is tantalising, since, in the context of the REL model, it may indicate that either the blue wing of the $\mathrm{N}$ line or the red wing of the $\mathrm{O}$ line are responding to the flares, which would be an important physical constraint on models of the emission region. However the current statistical significance of the effect is low. Confirmation of these changes with higher signal to noise data will be important.

Clearly more work is required before we fully understand the low energy X-ray spectrum of Mrk 766 and similar objects. On the observational side, there are a number of directions for future research. The variations in the profile of the lines, if that is what they are, with source flux are at the limits of detection in the current RGS data. Confirmation in other observations would provide an important physical constraint on potential models. We intend to examine the issue of spectral variability in Mrk 766 in more detail in a future publication, including consideration of the EPIC data. Similarly, a physical model of the warm absorber gas could be valuable in distinguishing which features come from the warm gas and which have other causes. It is encouraging, however, that the detail revealed by the current RGS data, coupled with the high throughput and energy coverage of the EPIC cameras, gives us real discriminating power. We have shown that the REL model is an elegant and relatively simple description of the $0.3-10 \mathrm{keV}$ spectrum of Mrk 766 . The physical processes that might produce such lines clearly demand further consideration as a mechanism to account for the spectrum of this and other Seyfert galaxies.

We thank Ted Brookings (UCSB) and Jelle Kaastra (SRON) for their work in developing the IMP and SPEX spectral fitting codes used in this analysis. 


\section{REFERENCES}

Ballantyne, D. R., \& Fabian, A. C. 2001, MNRAS 328, L11.

Ballantyne, D. R., Ross, R. R., \& Fabian, A. C. 2002, MNRAS (in press)astro-ph/0202148

Behar, E., Sako, M., \& Kahn, S. M., 2001 Ap.J. , 563, 497.

Blustin, A.J., Branduardi-Raymont, G., Behar, E., Kaastra, J.S., Kahn, S.M., Page, M.J., Sako, M., \& Steenbrugge, K.C., 2002 A\&A (in press: astro-ph 0206316).

Branduardi-Raymont, G., Sako, M., Kahn, S., Brinkman, A. C., Kaastra, J. S., \& Page, M. J. 2001 A\&A 365, L140.

den Herder, J.W., et al. 2001 A\&A 365, L7.

Kinkhabwala A., et al. 2002, ApJ, in press, astro-ph 0203290

Komossa, S., \& Bade, N., 1998, A\&A 331, L49.

Laor, A. 1991, ApJ, 376, 90.

Lee, J.C., et al. 2001 ApJ, 554, L13.

Lubinski, P., \& Zdziarski, A. A. 2001 MNRAS 323, L37.

Magdziarz \& Zdziarski, A. A. 1995, MNRAS, 273, 837.

Mason, K. O., et al. 2001 A\&A 365, L36.

McLaughlin, B. M., \& Kirby, K. P. 1998, J. Phys. B: At. Mol. Opt. Phys., 31, 4991.

Page, M.J., et al. 2001 A\&A 365, L152.

Reynolds, C., Ward, M. J., Fabian, A. C., \& Celotti, A. 1997, 291, 403.

Sako, M., et al. 2001 A\&A 365, L168.

Sako, M., et al. 2002 Ap.J. (in press).

Strueder, L., et al. 2001 A\&A 365, L18.

Tanaka, Y., et al. 1995 Nature 375, 659.

Turner, M.J.L., et al. 2001 A\&A 365, L27.

Verner, D.S. , Verner, E.M. \& Ferland, G.J. 1996a, Atomic Data Nucl. Data Tables, 64, 1. 
Verner, D.A., Ferland, G.J., Korista, K.T., \& Yakovlev, D.G., 1996, Ap.J., 465, 487.

Walter, R. \& Fink H. H. 1993, A\&A 274, 105.

Zycki P., \& Czerny, A., 1994, MNRAS, 266, 653. 